$81-2-70$

TAPEITSCHES ELEKTRONEN-SYNCHROTRON

DESY $80 / 115$

November 1980

MUON-NUCLEON SCATTERING, QCD RELATED EXPERIMENTAL RESULTS

by

F. W. Brasse

CERN, Geneva

and

Deutsches Elektronen-Synchrotron DESY, Hamburg

NOTKESTRASSE $85 \cdot 2$ HAMBURG 52 
DESY behält sich alle Rechte für den Fall der Schutzrechtserteilung und für die wirtschaftliche Verwertung der in diesem Bericht enthaltenen Informationen vor.

DESY reserves all rights for commercial use of information included in this report, especially in case of apply for or grant of patents.

To be sure that your preprints are promptly included in the HIGH ENERGY PHYSICS INDEX, send them to the following address ( if possible by air mail) :

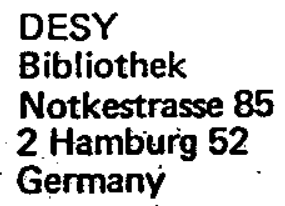


DESY $80 / 115$

November 1980

MUON-NUCLEON SCATTERING,

QCD RELATED EXPERIMENTAL RESULTS ${ }^{*}$ )

\author{
F.W. Brasse \\ CERN, Geneva, and \\ DESY, Hamburg
}

*) Talk presented at the International Summer Institute on Theoretical Physics, Bad Honnef, 1980. 
Recent results from muon-nucleon scattering on the structure function $F_{2}$, on multi-muon production and on the $\left\langle\mathrm{p}_{\mathrm{T}}^{2}\right\rangle$ of charged hadrons produced in the deep inelastic interaction are compared with QCD. 
MUON-NUCLEON SCATTERING,

QCD RELATED EXPERIMENTAL RESULTS

\author{
F.W. Brasse \\ DESY, Hamburg and \\ CERN, Geneva.
}

\title{
INTRODUCTION
}

Deep inelastic scattering of charged leptons on nucleons had lead with the observation of scaling of the structure functions to the quark parton picture of nucleons. With improved accuracy of the measurements and an increased scale of $Q^{2}$ in the scattering exper $i-$ ments violation of scaling was found giving an important basis for the new strong interaction theory, the Quantum Chromo Dynamics. It seems now that charged lepton nucleon scattering is entering a third phase in the understanding of hadronic interactions by actually testing quantitatively predictions of QCD. The muon beam at the CERN SPS with high intensity. and high energy makes it possible to conbine high accuracy with a large scale of energy and momentum transfer. Furthermore, not only the behaviour of structure functions can be compared with the theory but also particle production via detected hadrons or additional muons.

The European Muon Collaboration (EMC) has carried out a series of experiments which gave results at the same time for all areas mentioned above. I will concentrate the discussion therefore on results from this collaboration but will also refer to other experiments.

\section{MEASUREMENT OF $\dot{F}_{2}$, ITS SCALING VIOLATION AND QCD ANALYSIS}

Before discussing the experimental results it is worthwile to get an idea about the tools of measurements. Fig. 1 shows the apparatus of the EMC in an idealised three-dimensional view. The incoming muon beam impinges on a target, which had been for the measurements reported here either a $6 \mathrm{~m}$ liquid hydrogen target or a $2.0 \mathrm{~kg} / \mathrm{cm}^{2}$ iron 


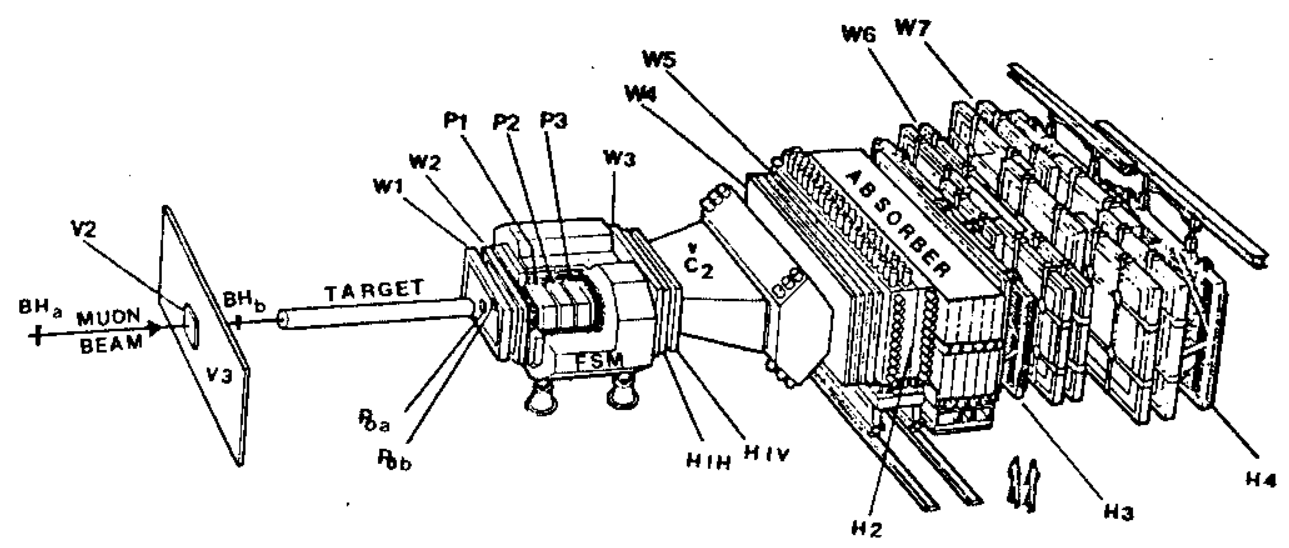

Fig. 1. The spectrometer of the EMC.

target interleaved with scintillators (STAC target) to measure the total hadronic or electromagnetic energy produced in the scattering process. The four momentum of charged particles leaving the target is determined by a large air gap magnet (FSM) and a set of drift (W1-5) and proportional (PO-3) chambers. Hadrons are stopped in the absorber so that muons are identified behind it by reconstructing their tracks in another set of large drift chambers (w6-7). Large hodoscopes are used for triggering ( $\mathrm{HI}-4)$ and for vetoing (VI-3) against halo muons. Some identification of charged hadrons is achieved by a Cerenkov counter $\left(\mathrm{C}_{2}\right)$ and of neutrals by a calorimeter $\left(\mathrm{H}_{2}\right)$.

Experiments on both targets have been carried out with different energies of the primary muon beam reaching from 120 to $280 \mathrm{GeV}$. For the measurement of structure functions this provides the largest possible coverage of the $Q^{2}, x$ olane with good overlap in this region among the data with different energies. Furthermore from the difference of the cross sections at a given $Q^{2}, x$ point measured with different primary energies it is possible to determine $R$, the ratio of the longitudinal to transverse virtual photon cross section. Results on this value have not been obtained up to now from the EMC measurements; therefore the assumption $R=0.2$ as an average result at lower $Q^{2} i$ has been made for the determination of $F_{2}$.

The structure function $\mathrm{F}_{2}$ as obtained from measurements on the iron target and from combining three different primary energies is shown in fig. 2 as a function of $Q^{2}$ for different bins in $x$. At small values of $x$ the structure function $F_{2}$ clearly rises with increasing $Q^{2}$ whereas at large $x$ it falls definitely. At small $x$ the upper limit in $Q^{2}$ is given. by the primary energy whereas in the highest $x$ bin is still dictated by statistics. The amount of scaling violation is better visible in $f i g .3$, where the value $b=\mathrm{d} l \mathrm{nF}_{2} / \mathrm{dQ}^{2}$ as fitted to the different $x$ bins is shown as a function of $x$. For $x \geqslant 0.25$ results on $F_{2}$ in the higher $Q^{2}$ region have been obtained also by the 

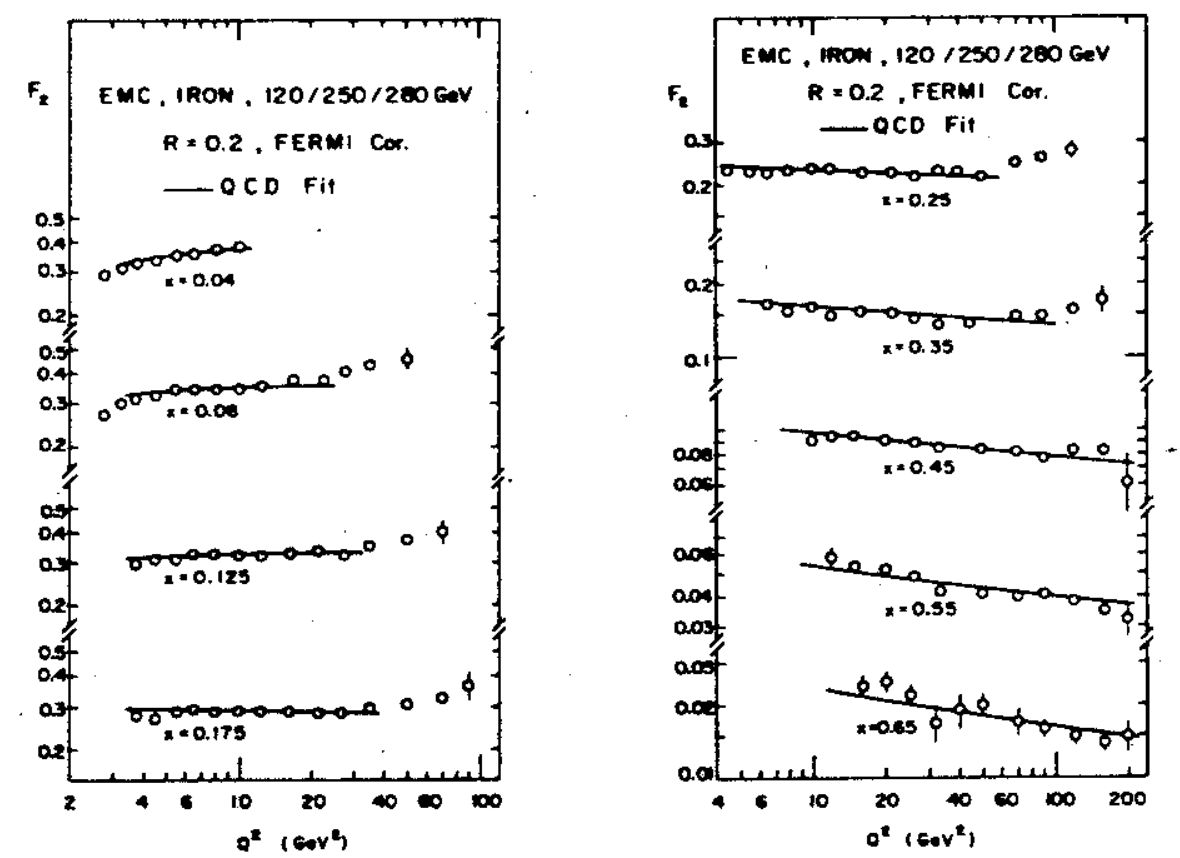

Fig. 2. $\mathrm{F}_{2}$ as measured by $\mathrm{EMC}^{2}$ on iron.
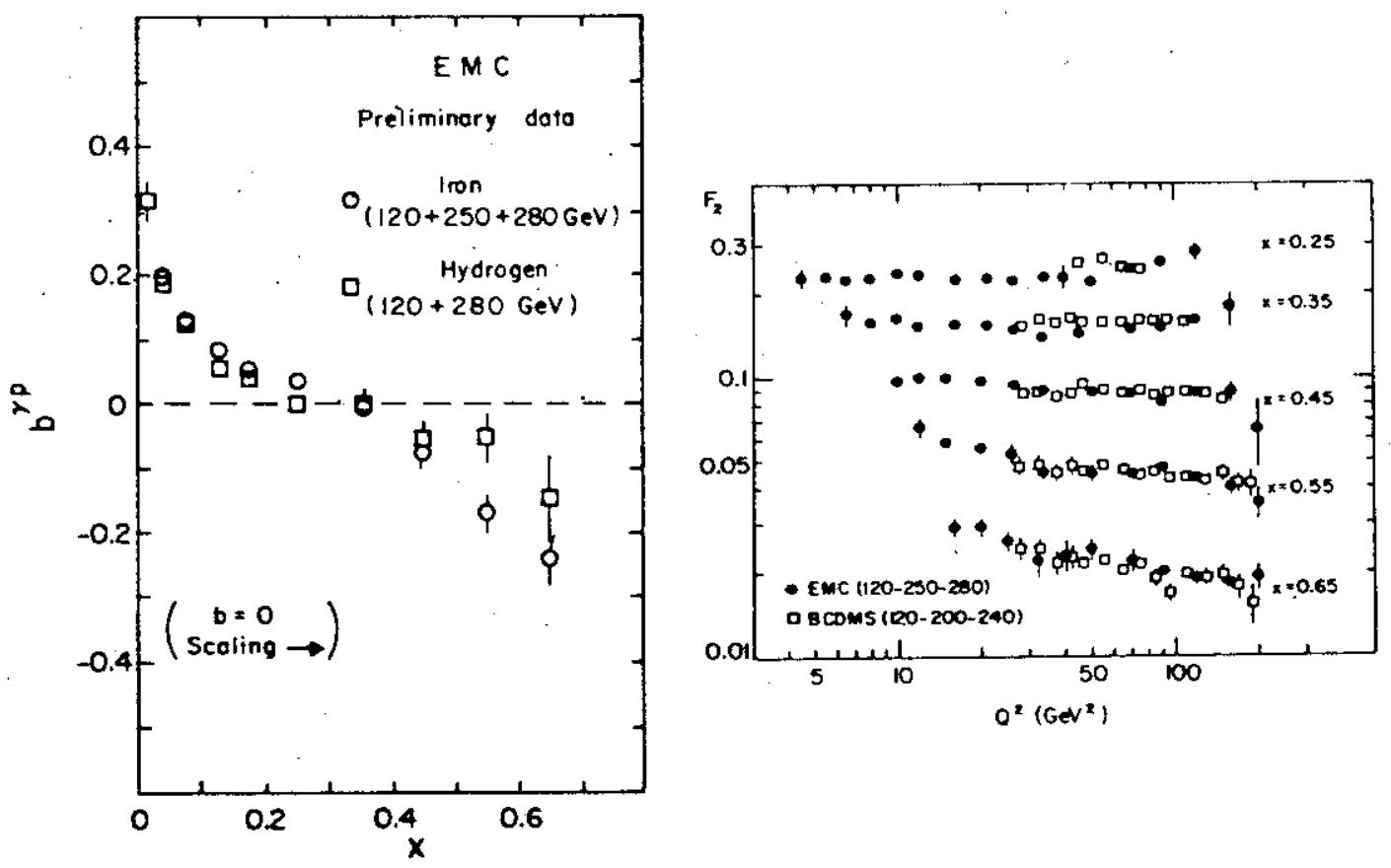

Fig. 3. The scaling violation parameter $b$.

Fig. 4. $\mathrm{F}_{2}$ measured by $\mathrm{EMC}^{2}$ and $\mathrm{BCDMS}^{3}$. 


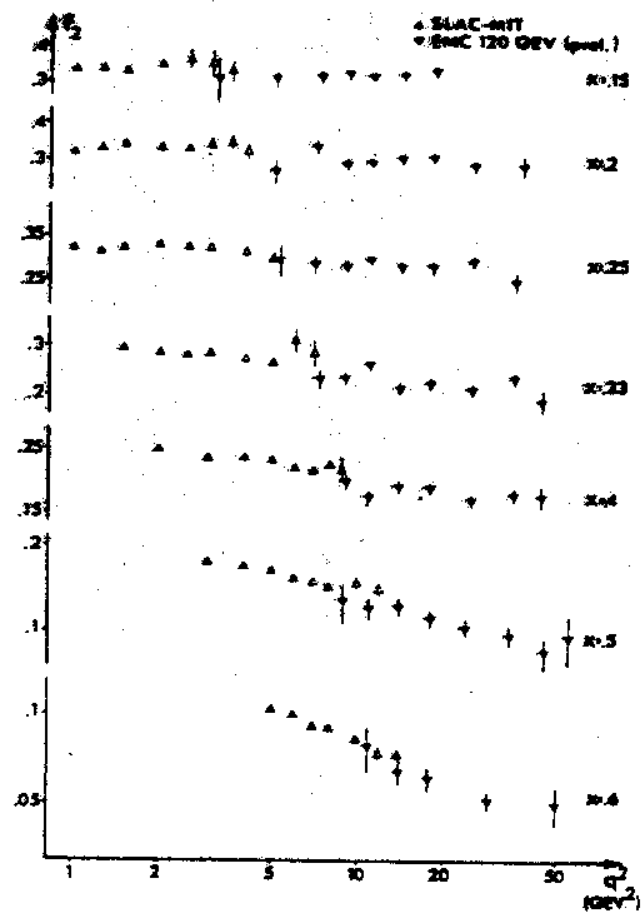

Fig. 5. $F_{2}$ as measured by Exic on hydrogen at Iow energy compared with SIAC datal.

BCDWS $^{3}$ group at Cbin, using a carbon target. Their results as a combination of three different energies are compared in fig. 4 with the results from the EMC on the iron target. In this case both data sets are not corrected for Fermi motion, whereas in fig. 2 this correction is in. The agreement between the two sets of structure functions is remarkable.

To reach as low $Q^{2}$ as possible the lowest energy was $120 \mathrm{GeV}$ for both targets. For the hydrogen target the results from $\mathrm{EMC}^{4}$ are shown in fig. 5 and are compared with data from SLAC ${ }^{1}$. Both data sets join on to each other rather well although a difference in mormalisation up to $10 \%$ is not excluded.

The low energy $\mathrm{F}_{2}$ data $(120 \mathrm{GeV})$ and the high energy data on hydrogen $(280 \mathrm{GeV}$ ) of the EMC are shown together in fig. 6. The agreement in the overlapping regions of $Q^{2}$ is excellent. The general behaviour of $F_{2}$ as a function of $x$ and $Q^{2}$ is the same as found on the iron target. It is represented by the slope paraneter $b$ shown in fig. 3 . Also seen in $f i g .6$ is the change of $F_{2}$ if $R$ is equal to zero instead of 0.2 . At values of $x$ up to 0.08 the changes are very noticeable and can be as large as $12 \%$.

The curves in figs. 2 and 6 are the result of a QCD analysis ${ }^{5}$. Two methods have been applied, which both make direct use of the Altarelli-Parisi equations. The basic advantage is, compared to a moment analysis, that it is not necessary to extrapolate the 

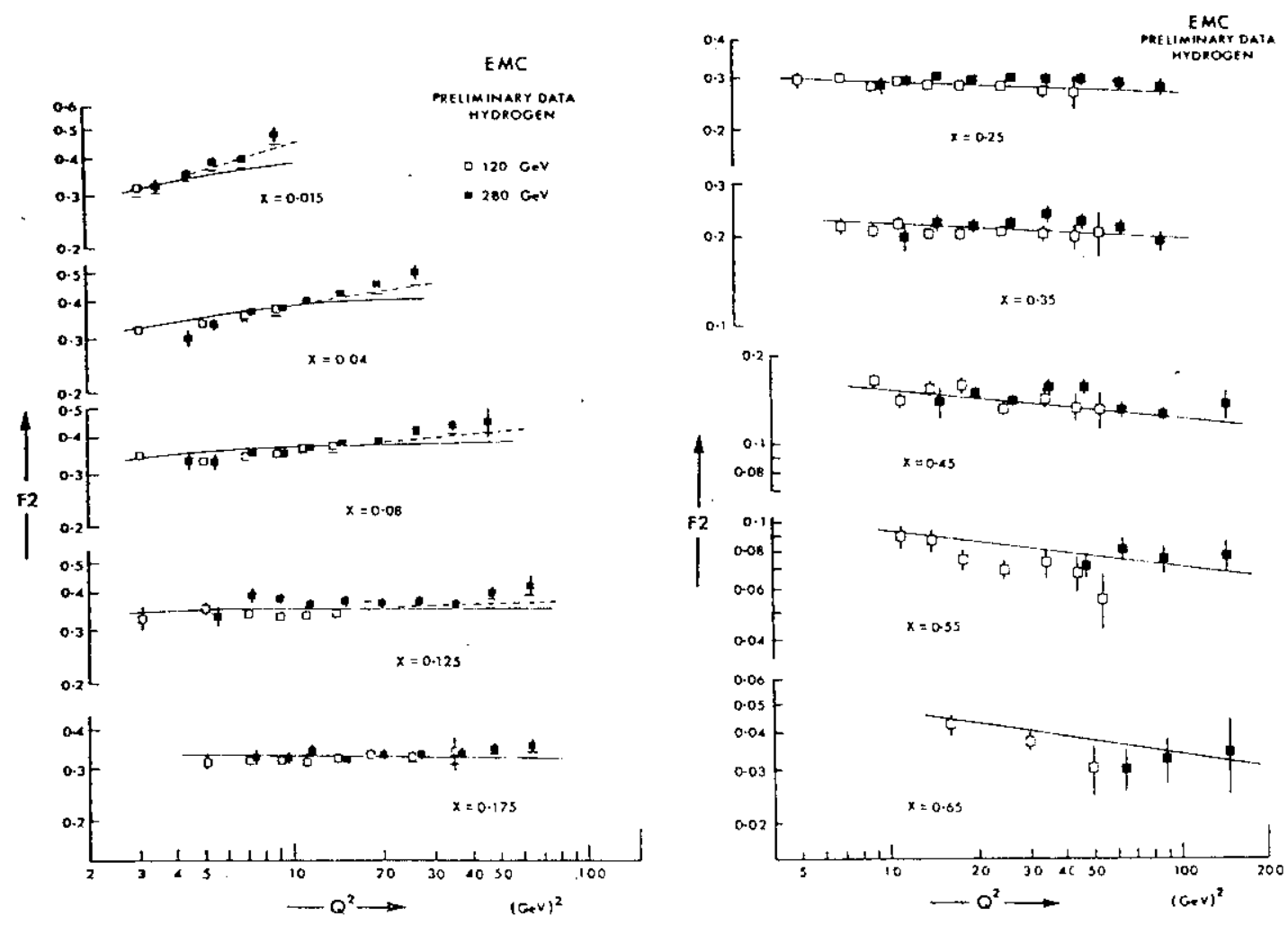

Fig. 6. $\mathrm{F}_{2}$ as measured by EMC on hydrogen at low and high energy. (- QCD fit; ---fit + charm production; I data with $\mathrm{R}=0$ )

measured $F_{2}\left(x, Q^{2}\right)$ into low $x$ regions, which are unaccessible to the experiment. One of the two methods used by the EMC has been developed by Gonzales-Arroyo et $\mathrm{al}^{7}$. It was restricted to the non singlet part of the structure function and to leading order. As $F_{2}$ in $\mu p$ scattering has contributions at small $x$ from the sea (being a singlet part), $x$ was restricted to $x \geqslant 0.25$, where the sea contributions are very smal $1^{8}$. For the parameterization of $\mathrm{F}_{2}$ at a fixed $\mathrm{Q}_{0}^{2}$ the relation

$$
F_{2}\left(x, Q_{0}^{2}\right)=A x^{\alpha}(1-x)^{\beta}(1-\gamma x)
$$

was used. All data in the region defined above were then taken to determine $\alpha, \beta, \gamma$ and the scale parameter $\Lambda$, which is related to the running coupling constant $\alpha_{s}$ in leading ordex by

$$
\alpha_{s}=\frac{4 \pi}{\beta_{0} \log \left(Q^{2} / \Lambda^{2}\right)}, \quad B_{0}=\frac{33-2 n_{f}}{3} \text {. }
$$

The normalisation factor A was either fixed by sum rule or as a test left free. The results did not depend on either choice. Also the choice of $Q_{0}^{2}\left(2,7\right.$ and $20 \mathrm{GeV}^{2}$ were used) did not alter the fit parameters. The results on these parameters for the EMC data as well as for SLAC data are shown in table 1 . 
Table 1: Results from a QCD fit using the method of Gonzàles-Arroyo et al.(for $\left.Q_{0}^{2}=2 \mathrm{GeV}^{2}\right)^{7}$.

\begin{tabular}{|c|r|c|c|c|c|}
\hline Data & $\Lambda(\mathrm{MeV})$ & $\alpha$ & $\beta$ & $\gamma$ & $\chi^{2} / \mathrm{NDF}$ \\
\hline EMC $\mu \mathrm{Fe}$ & $<100$ & 0.74 & 3.40 & 0.59 & $83^{*} / 72$ \\
EMC $\mu \mathrm{p}$ & $<100$ & 0.71 & 3.20 & 0.23 & $85^{*} / 75$ \\
SLAC ep & 250 & 0.69 & 2.30 & 0.34 & $93^{*} / 39$ \\
SLAC ed & 340 & 0.65 & 2.46 & 0.22 & $18 / 39$ \\
\hline
\end{tabular}

$\left.{ }^{*}\right)$ only statistical errors are included.

The second method used by the EMC is the one of Abbott et al ${ }^{9}$. This analysis was not restricted to the non-singlet part, for which the parameters of Abbott et al of $F_{2}^{N S}\left(x, Q_{0}^{2}\right)$ were used. The parameterization of $F\left\{\left(x, Q_{0}^{2}\right)\right.$ and $\Lambda$ were left free. The results of these fits in terms of $\Lambda$ and $F_{2}$ itself for $x>0.25$ were practically identical to those obtained by the other method. In addition a fit to the data was obtained for $x<0.25$. In figs 2 and 6 the results from this method are compared with the data for both targets. At sma1l $x$ the agreement between the fit and the data is clearly not as good as at medium and large $x$, especially for the smallest $x$ bin $x=0.015$ on the hydrogen target. For a fixed value of $x$ one is crossing the charm production threshold with increasing $Q^{2}$, getting a contribution to $F_{2}$ which does not scale and which is not covered by the QCD calculation, at least not near threshold. The rg-fusion model used successfully to explain the dimuon events in terms of charm production (see below) has been taken to calculate this contribution. The result is shown in fig. 6, indicating that a large fraction of the difference between fit and data can be accounted for by charm production. In addition to the stronger rise of the data with $Q^{2}$ up to $x=0.15$ on the hydrogen target the iron data show a rise with $Q^{2}$ up to $x=0.35$ which starts at a certain high value of $Q^{2}$ (i.e. for values of $y=v / E$ close to the cut value of $0.85-0.90$ applied to the data). This behaviour of the data is not understood. Although radiative corrections have been applied there may be the question how reliable the calculations are for a heavy target at large values of $y$.

Table 1 clearly shows that the higher $Q^{2}$ data from EMC give a smaller value for $\Lambda$ than the lower $Q^{2}$ data from SLAC. The error on $\Lambda$ as given by the EMC is about $100 \mathrm{MeV}$ (but excluding $\Lambda=0$ ) and is mainly coming from systematic errors on the relative normalisation of data sets from different primary energies and from uncertainties on the energy calibration of the primary muon. The influence of the SLAC data on $\Lambda$ has also been demonstrated by the MSUF collaboration Ball et al 10 . Their results for $F_{2}$ on iron are shown together with the SLAC ed results in fig. 7. There is no overlap between the two 
different data sets. But nevertheless a QCD fit has been made by Ball et al., using also the method of Abbott et al., to the combined data sets giving $\Lambda=0.5 \pm 0.2 \mathrm{GeV}$. The result for the MSUF data alone is $\Lambda=0.1 \pm 0.05 \mathrm{GeV}$.

One possibility to explain the difference on $\Lambda$ between SLAC and EMC is the inclusion of higher twist terms, which can give a larger contribution at low $Q^{2}$. The fit for $x \geqslant 0.25$ to the SLAC results is dominated by the $Q^{2}$ range $1-5 \mathrm{GeV}^{2}$, whereas that to the EMC data by $5-80 \mathrm{GeV}^{2}$ on the hydrogen target and $5-150 \mathrm{GeV}^{2}$ on the iron target. An attempt ${ }^{5}$ has been made to quantify the higher twist contribution by the addition of a term proportional to $1 / Q^{2}$ to the parameterization of $F_{2}$ for the $Q C D$ analysis. The result is rather satisfactory giving then also $\Lambda=100 \mathrm{MeV}$ for a reasonable small positive contribution from $1 / Q^{2}$ term. There is also a difference in the result for $\Lambda$ between the muon scattering experiments as given above and neutrino interactions as published so far by the CDHS collaboration ${ }^{11}$. It is doubtful that this difference could also be explained by higher twist terms as the kinematic ranges and statistical weights are rather similar. On the other hand the analysis of the preliminary new data from $\mathrm{CDHS}^{12}$, using $\mathrm{xF}_{3}$, gives a value $\Lambda=0.3 \pm 0.15$, which is not inconsistent with the result from the muon experiments.

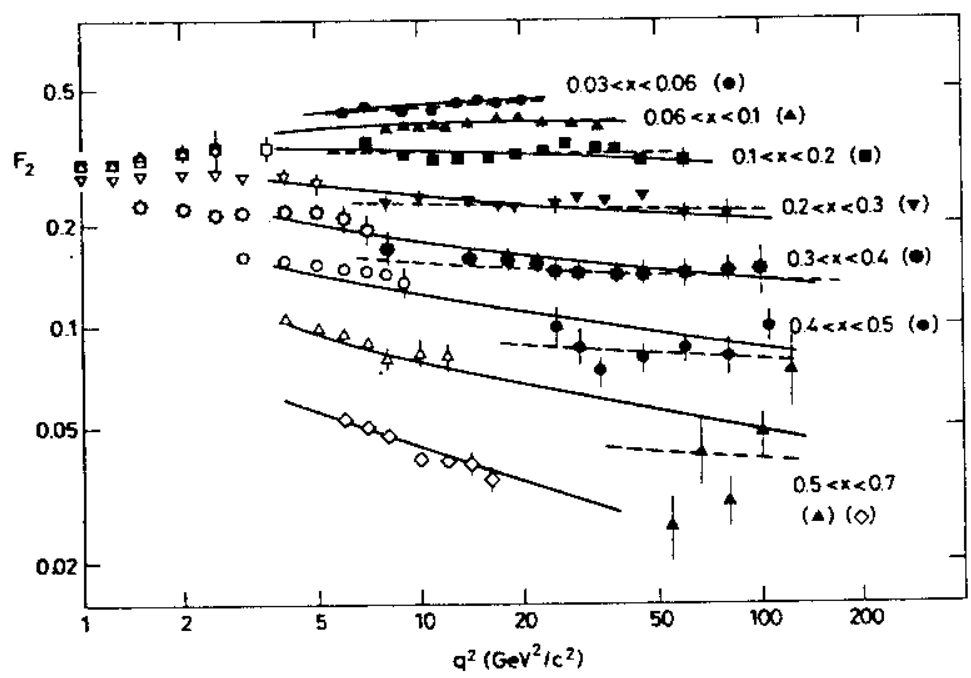

Fig. 7. $\mathrm{F}_{2}$ as measured by $\mathrm{MSUF}^{10}$ on iron compared with SLAC ed datal and with QCD fits. 


\section{MULTI-MUON PRODUCTION}

Since the discovery of charm it is known that open charm production should be a rich source of multi-muons and that by measuring them the production mechanism could be studied. First experimental results had been delivered by the MSUF experiments ${ }^{3}$ and could qualitatively be explained by $D \bar{D}$ production. Now with the existence of more precise data a quantitative comparison with models can be made.

The favoured model now is the photon gluon fusion model ${ }^{14}$, the diagram of which is shown in fig. 8. Dynamically the model says that, contrary to the same diagram for light quarks, the momentum coming in with the virtual photon is shared between both c quarks. Either one or both of the $D$ mesons decay semi-leptonically to have either two or three muons in total in the final state. The signature of this process is wissing energy carried away by the neutrinos. The EMC has analysed these events from data using the iron target, where the

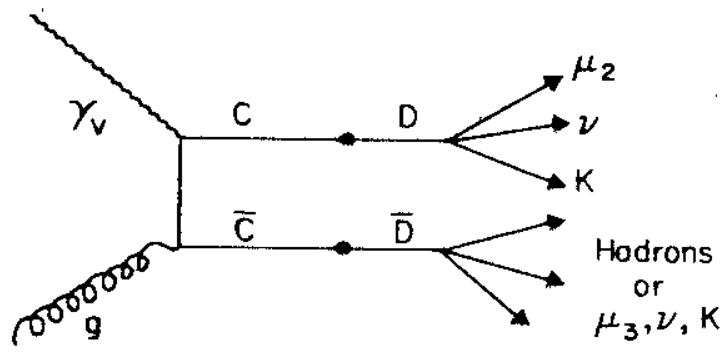

Fig. 8. The photon-gluon fusion diagram for $c \bar{c}$ production with one or two muons in the final state.

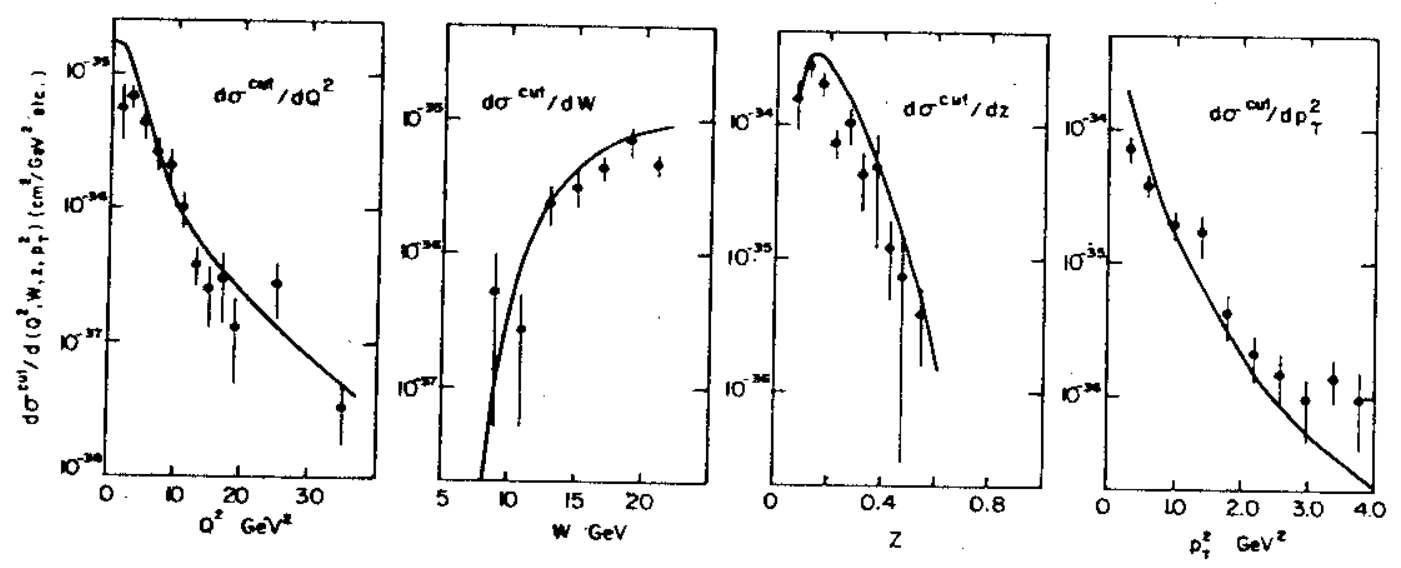

Fig. 9. Di-muon results from $E M C^{15}$ compared with $\mathrm{Yg}$-fusion mode1. 
missing energy was determined by the STAC:

$$
E_{\text {Miss }}=E_{\text {Beam }}-E_{S T A C}-E_{\mu_{1}}-E_{\mu_{2}}\left(-E_{\mu_{3}}\right) \text {. }
$$
Here E STAC $_{\text {is the energy deposited in the STAC. Results for dimuons }}{ }^{15}$
are shown in fig. 9 in the form of cross sections for the sensitive part of the apparatus ( $d \sigma_{\text {cut }}$ ). The main background to the process comes from $\pi$ and $\mathrm{K}$-decay ( are the results from the $\mathrm{rg}$-fusion model calculation as an absolute prediction. There is remarkably good agreement in the shape of the distribution as well as in the absolute height. Similarly good agreement was reached by $\mathrm{Clark}$ et $21 . .^{18}$ between their di-muon data taken at FNal and the $\gamma \mathrm{g}$-fusion model.

Also tri-muon data have been analysed by the EMC ${ }^{16}$ using inelastic events in the mass range $1.0<\mathrm{m}_{\mu \mu}<2.5 \mathrm{GeV}^{2}$ as indicated in fig. 10. The inelastic events were selected by requiring $E_{\text {TAC }}>5 \mathrm{GeV}$. Furthermore inelastic events with only electromagnetic showers were suppressed by using the information on the shower length from the STAC. To do this the energy threshold had to be raised from 5 to $20 \mathrm{GeV}$. Again the results in the form of do cut are compared with the $\mathrm{rg}$-fusion model ( $\mathrm{fig} .11$ ) and are found to be in good agreement.

The input to the $\gamma g$-fusion model $\left(\Lambda=0.5 \mathrm{GeV}, \mathrm{m}_{\mathrm{c}}=1.5 \mathrm{GeV}\right.$, $x G(x)=3(1-x)^{5}, D_{c}^{D}(z)=$ constant) has not been varied so far. As in the case of EMC only 15\% of all high energy iron data up to now are in the multi- $\mu$ results, with the full statistics a much more quantitative comparison with the model will be possible and, assuming the mode1 is correct, the best values for the QCD input parameters fitting the data will be found.

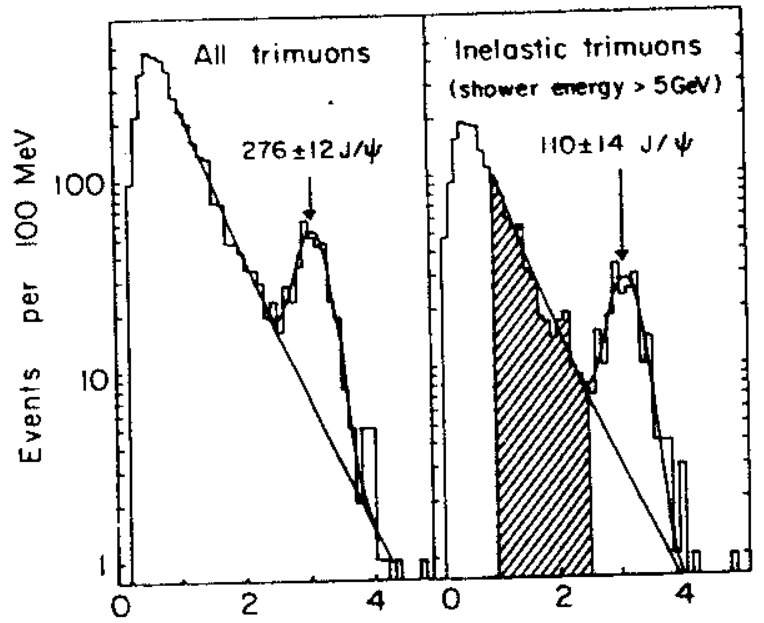

Fig. 10. Tri-muons, spectra of the mass (GeV) for the $\left(\mu_{2} \mu_{3}\right)$ pair. 

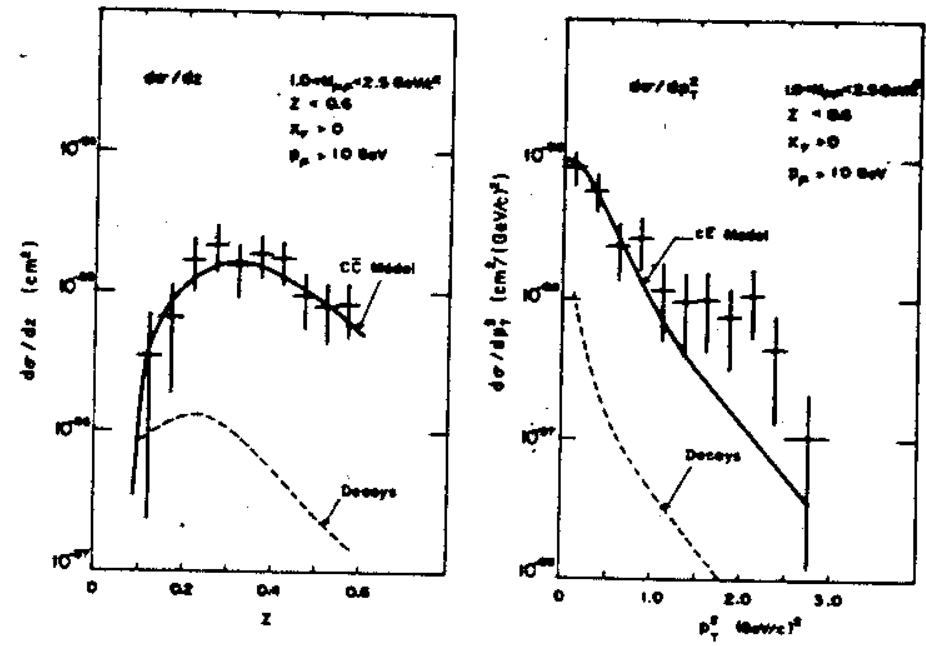

Fig. 11. Tri-muon results from EMC ${ }^{16}$ compared with Yg-fusion model.

Already elastic J/ $\psi$ production (see fig. 10) in muon nucleon scattering ${ }^{3}, 18$ is providing information about the gluon distribution. The measured $Q^{2}$ and $P_{T}$ dependences have been used to determine $d \sigma / d t$ at $Q^{2}=0$ as a function of the energy $\nu$ of the photons. These cross sections combined with those determined from real photoproduction ${ }^{19}$ at lower energies as well as the individual distributions $\mathrm{d} \sigma / \mathrm{dQ}^{2}$ and $\mathrm{d} \sigma / \mathrm{dp}_{\mathrm{T}}^{2}$ have been compared with the $\mathrm{rg}$-fusion model and are found to be in good agreement ${ }^{2}$. However, some arbitrariness exists in the absolute normalisation for the model. The $\nu$-dependence of $\sigma(\gamma \mathrm{N} \rightarrow \mathrm{J} / \psi \mathrm{N})$ has been used to calculate the gluon distribution $G(x)^{20,21}$. The comparison with two curves (fig. 12) shows that the data clearly support $x G(x)=3(1-x)^{5}$, the distribution which has been used throughout all yg-fusion model calculations presented here.

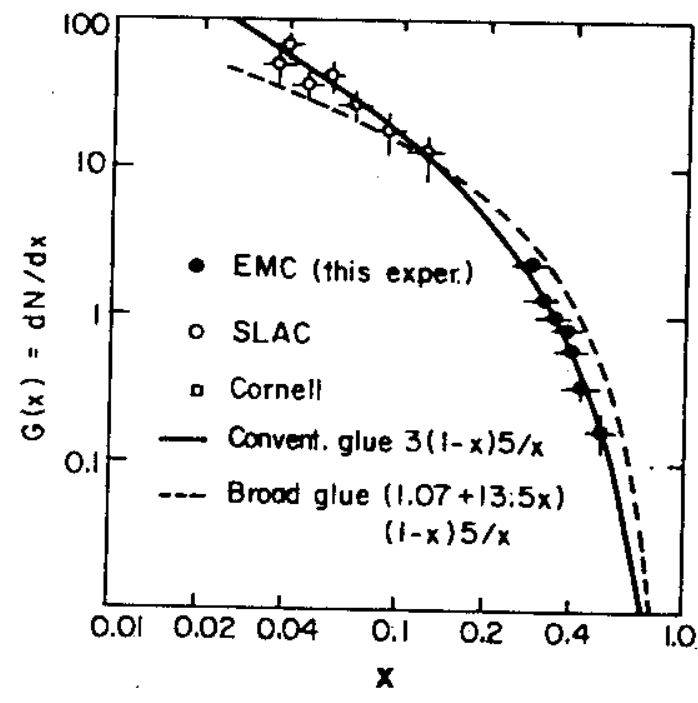

Fig. 12. The gluon distribution as calculated from elastic $J / \psi$ production and compared with two curves as used in models. 
The European Muon Collaboration has also deduced results on inelastic $\mathrm{J} / \psi$ production $\left(\mathrm{E}_{\mathrm{STAC}}>5 \mathrm{GeV}\right.$ ), giving perhaps another piece of information on the coupling of the $c \bar{c}$ system to the nucleon. $110 \pm 14$ events after background substraction were found inside the cut regions of $60<\nu<180 \mathrm{GeV}$ and $z>0.3$.

The corresponding number of elastic $J / \psi$ events in the energy range $50<\nu<180 \mathrm{GeV}$ was 166. A possible source of inelastic J/ $\psi$ 's is the production of higher charmonia states such as $\psi^{\prime}$ and $\chi$, decaying into $\mathrm{J} / \psi$. However at most $40 \%$ of the observed rate can be accounted for in this way. Furthermore, applying the $\mathrm{\gamma g}$-fusion model to the production of these states, the resulting $z$ and $\nu$ dependencies of the cross section do not agree with the data ${ }^{22}$. Another possible source is Dre11-Yan type production which however has been estimated to be small.

Duke and Owens ${ }^{23}$ have done calculations on the basis of the $\gamma$-fusion diagram but taking into account also higher order QCD diagrams such as the additional emission of soft and hard gluons, providing the inelasticity. Their results are compared in fig. 13 with the data in terms of $\mathrm{p}_{\mathrm{T}}$ and $\mathrm{z}$ distributions. The overall normalisation for the total (elastic and inelastic) production is free. The agreement is very encouraging, and if the calculations are further supported by more data, this would be a direct measurement of higher order QCD graphs and could have some impact on the question, to what extent higher orders of QCD are important for the interpretation of $\mathrm{F}_{2}$.
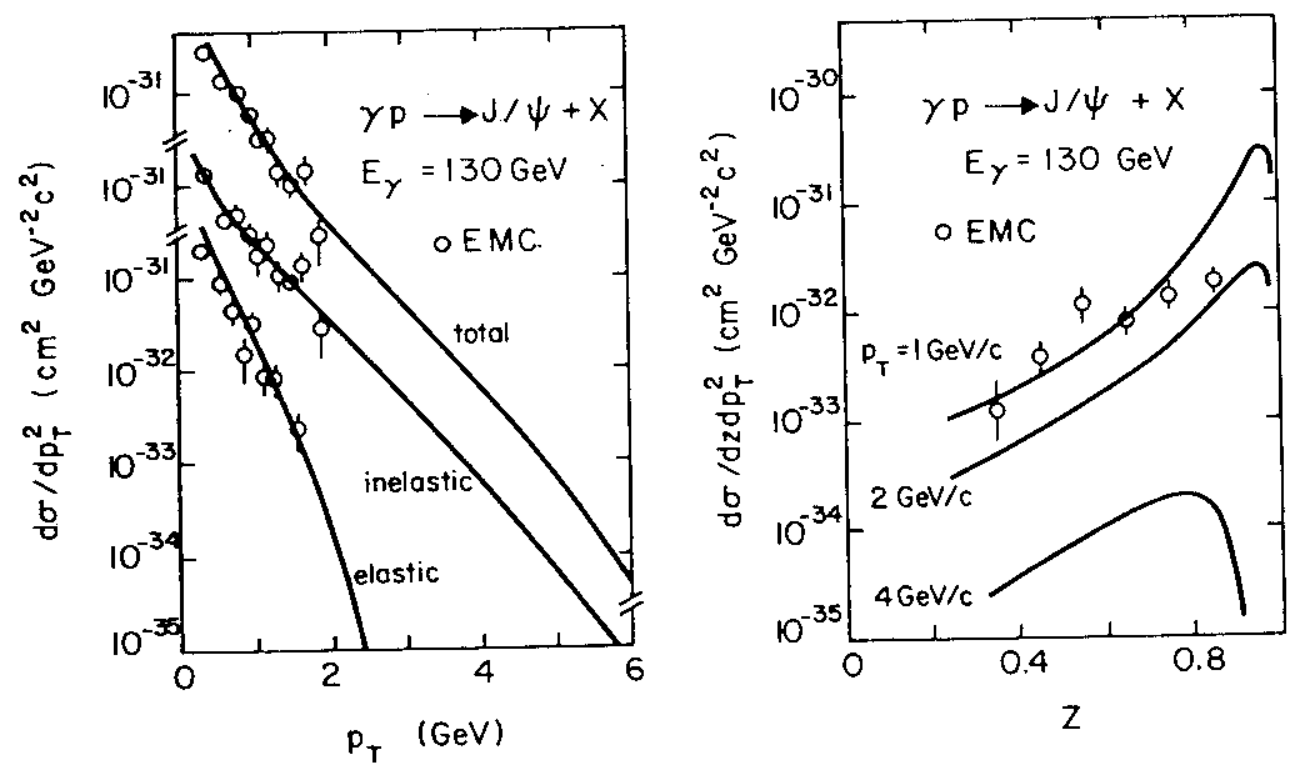

Fig. 13. E1astic ${ }^{17}$ and inelastic $J / \psi$ measurements ${ }^{22}$ compared with higher order QCD calculations ${ }^{23}$. 


\section{TRANSVERSE MOMENTUM OF HADRONS}

The emission of hard gluons and the production of $q \vec{q}$ pairs according to the diagrams in fig. 14 lead to a broadening of the hadron distributions, which means an increase of the average transverse momentum of the forward going hadrons. The general belief therefore is that $\left\langle p_{T}^{2}\right\rangle$ is composed of three parts: one coming from the fragmentation of quarks, another from the intrinsic $k_{T}$ of the quarks (Fermi motion) and the third one being the QCD part as described bef 2 re. Hadron production in muon scattering has been studied $^{24,25}$ to separate the different contributions. This is in principle possible using the different dependencies on $w$, the total hadronic energy, and on $z=E_{h} / v$. Whereas the QCD part is mainly depending on $W$, the part of $\left\langle\mathrm{p}_{\mathrm{T}}^{2}\right\rangle$ coming from the intrinsic $k_{T}$ is proportional to $z^{2}$. The fragmentation part should neither depend strongly on Wor on $z$.

In fig. $15\left\langle p_{T}^{2}\right\rangle$ from the EMC data ${ }^{25}$ is shown as a function of $W^{2}$ for different bins of $z$. A strong increase with $W^{2}$ as well as with $z$ is seen. The QCD part has been calculated ${ }^{26}$ in first order of $\alpha_{s}$ in absolute agnitude as a function of $w^{2}$ for the different bins of $z$. This result has been fitted to the data leaving the nonperturbative part of $\left\langle p_{T}^{2}\right\rangle$ for each $z$ bin as a free parameter. The result of these fits is also given in fig. 15 showing that the $W^{2}$ dependence of the data is well represented by QCD. The $z$ dependence of $\left\langle p_{T}^{2}\right\rangle$ as determined experimentally is given in fig. 16 showing the very stroag seagull effect. To extract a value on $\left\langle k_{T}^{2}\right\rangle$, (curve I) has been calculated via Monte Carlo, using standard methods with a cascade model with an exponential $p_{T}^{2}$ distribution

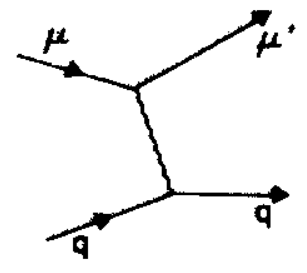

a) zero-order diogram

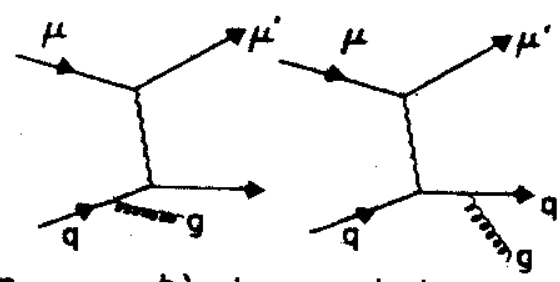

b) gluon emission

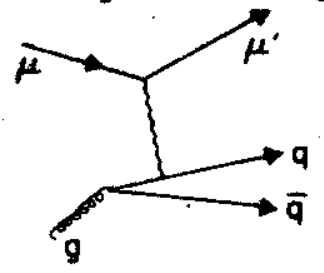

c) quark-antiquark emission

Fig. 14. The zero and first order QCD diagrams. 


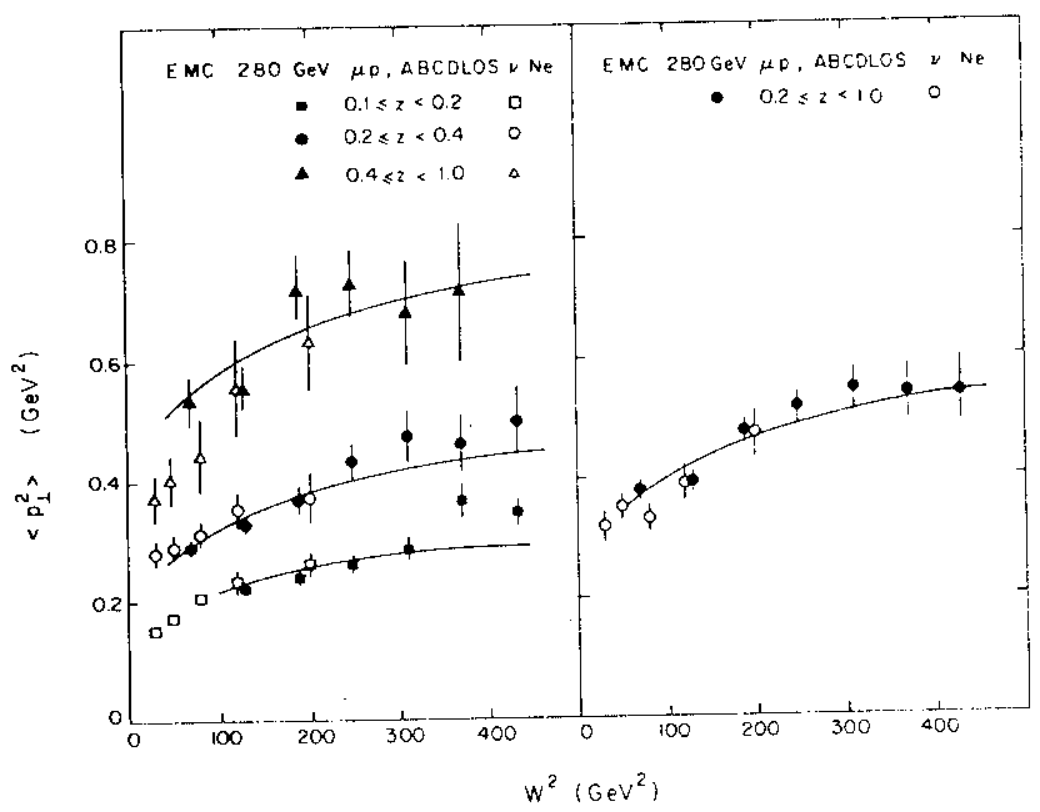

Fig. 15. The measured $\left\langle\mathrm{p}_{\mathrm{T}}^{2}\right\rangle$ as a function of $\mathrm{W}^{2}$ compared with the shape as calculated from QCD.

with $\sigma=0.35 \mathrm{GeV}$. A fit has then been made to the data (with $z^{2}>0.2$ ) including a term $\left\langle\mathrm{p}_{\mathrm{T}}^{2}\right\rangle=\mathrm{z}^{2} \cdot\left\langle\mathrm{k}_{\mathrm{T}}^{2}\right\rangle$ with $\left\langle\mathrm{k}_{\mathrm{T}}^{2}\right\rangle$ as the fit parameter. The overall functional dependence of the data is well described by this model (curve IV) and the result on $\left\langle k_{\mathrm{T}}^{2}\right\rangle$ is $0.63 \pm 0.10 \mathrm{GeV}^{2}$. A similar result has been obtained by the $\mathrm{DECO}$ experiment ${ }^{27}$ and recently also from

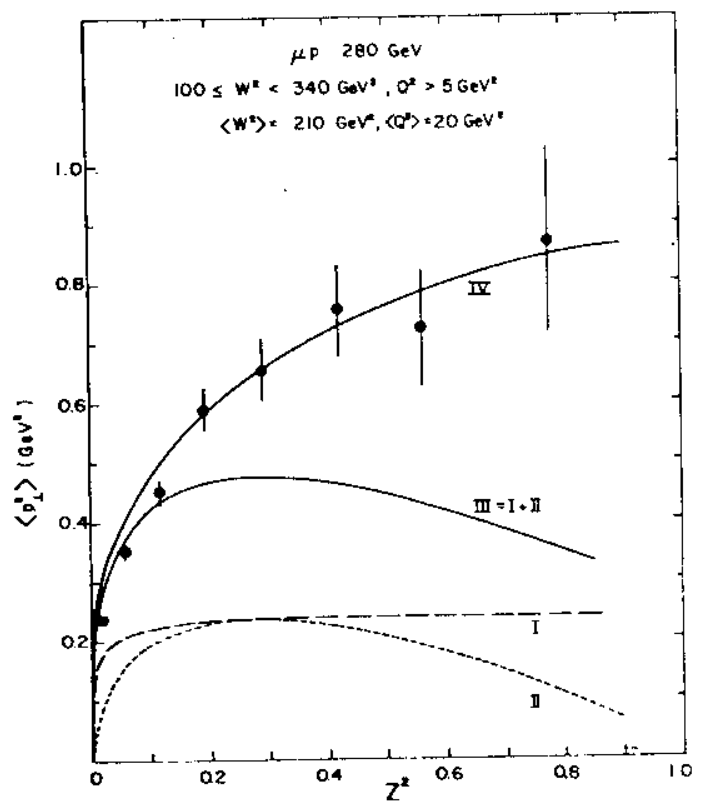

Fig. 16. The measured $\left\langle\mathrm{p}_{\mathrm{T}}^{2}\right\rangle$ vs. $z^{2}$. For the curves see text. 
di-muon data in neutrino interactions ${ }^{28}$.

Again, as for the study of the rg-fusion model, the QCD input parameters ( $\Lambda$, quark and gluon distributions) have not been varied. This clearly has to be done in the future to get a coherent picture. SUMMARY

In summary one can say that muon nucleon scattering is testing the thory of QCD along different lines in a very powerful way. The main results from the experiments are:

(a) $\mathrm{F}_{2}$ on iron and hydrogen over $4 \leqslant \mathrm{Q}^{2} \leqslant 200 \mathrm{GeV}^{2}$ shows clearly scaling violation. The scale parameter $\Lambda$ in leading order comes out to be about $100 \mathrm{MeV}$.

(b) The $\gamma g$-fusion mode1 (1. order QCD diagram) can explain all multi- $\mu$ data except the inelastic $J / \psi$ production.

(c) The $\left\langle p_{\mathrm{T}}^{2}\right\rangle$ behaviour of charged hadrons can well be described by QCD. In a simplified model $\left\langle\mathrm{k}_{\mathrm{T}}^{2}\right\rangle$ is $20.6 \mathrm{GeV}^{2}$.

\section{REFERENCES}

1. A. Bodek et a1., Phys. Rev. D20 (1979) 1471.

2. J.J. Aubert et al., "Measurement of the Nucleon structure Function $\mathrm{F}_{2}$ by Muon Iron Interactions at 280,250 and $120 \mathrm{GeV}^{\prime \prime}$, contribution to the Madison Conference 1980.

3. D. Bollini et a1., CERN-EP/80-133.

4. J.J. Aubert, et al., "Measurement of the Proton Structure Function $\mathrm{F}_{2}$ in Muon-Hydrogen Interactions at 280 and $120 \mathrm{GeV}^{\prime \prime}$, contribution to the Madison Conference 1980.

5. J.J. Aubert et al., "Scaling Violation in $\mu \mathrm{N}$ scattering and a Possible Interpretation", contribution to the Madison Conference
1980.

6. G. Altarelli and G. Parisi, Nucl. Phys. B126 (1977) 298.

7. A. Gonzàles-Arroyo et al., Nucl. Phys. B159 (1979) 512;

8. Nucl. Phys. B153 (1979) 161.

8. A.J. Buras and K.J.F. Gaemers, Nucl. Phys. BI32 (1978) 249. Recent preliminary results from $v$ interactions (CDHS collaboration, presented at the Madison Conference 1980) indicate,

9. L. Abbott and R.M. Barnett, SLAC-PUB at $x=0.25$ may not be small.

L. Abbott, SLAC-PUB 2325 (1979);

10. R. A. Ball, Phys. SLAC-PUB 2400 (1979).

10. R.C. Ball, Phys. Rev. Lett., to be published, MSU-CSL-80.

11. J.G.H. de Groot et al., Phys. Lett. 82B (1979) 456.

12. J.G.H. de Groot, "New Results on Neutrino Induced Charged Current Interactions", report at the Madison Conference 1980.

13. K.W. Chen, MSU-CSL-51 and Proceedings of the 1977 International Symposium on Lepton and Photon Interactions at High Energies, Hamburg;

D. Bauer et al., Phys. Rev. Lett. 43 (1979) 1551. 


\section{REFERENCES (Cont'd)}

14. J.P. Leveille and T. Weiler, Nucl. Phys. B147 (1979) 147.

15. J.J. Aubert et al., Phys. Lett. 94B (1980) 96, CERN-EP/80-61.

16. J.J. Aubert et a1., Phys. Lett. 94B (1980) 101, CERN-EP/80-62.

17. J.J. Aubert et a1., Phys. Lett. 89B (1980) 267, CERN-EP/79-140.

18. A.R. C1ark et a1., LBL-10747, 1980;

A.R. Clark et al., Phys. Rev. Lett. 43 (1979) 187.

19. R. Gittelmann et al., Phys. Rev. Lett. 36 (1975) 1616;

U. Camerini et al., Phys. Rev. Lett. 35 (1975) 483.

20. R.P. Mount, "Multimuon Production in $280 \mathrm{GeV} \mathrm{\mu ^{+ }}$ Iron Interactions" report at the Madison Conference 1980, also internal report $\mathrm{EMC} / 80 / 21$.

21. J.K. Davies, interna1 report EMC $/ 80 / 7$.

22. J.J. Aubert et al., CERN-EP/80-84.

23. D.W. Duke and J.F. Owens, FSU-HEP-800709 (1980).

24. C. Tao et al., Phys. Rev. Lett. 44 (1980) 1726.

25. J.J. Aubert et al., Phys. Lett. to be published, CERN-EP/80-119.

26. P. Kro11, private communication.

27. F. Janata, Proceedings of the EPS Conference, Geneva 1979, 775.

28. J. Knobloch, report at the Madison Conference 1980. 